\title{
A Seamless Handoff Scheme for IEEE 802.11 Wireless Networks
}

\author{
Yun-Sheng Yen \\ Department of Informatics \\ Fo Guang University \\ Ilan, Taiwan, R.O.C. \\ ysyen@mail.fgu.edu.tw
}

\author{
Ruay-Shiung Chang \\ Department of Computer Science \\ and Information Engineering \\ National Dong Hwa University \\ Hualien, Taiwan, R.O.C \\ rschang@mail.ndhu.edu.tw
}

\author{
Tsung-Yung $\mathrm{Wu}$ \\ Department of Computer Science \\ and Information Engineering \\ National Dong Hwa University \\ Hualien, Taiwan, R.O.C \\ cywu@ms01.dahan.edu.tw
}

\begin{abstract}
The advance of computer network technologies such as IEEE 802.11 Wireless Local Area Network enables users to connect to Internet almost anywhere. A mobile node is likely to move between different base stations while running applications. The IETF defines the Mobile IP (MIP) allowing mobile nodes to maintain their communication uninterrupted while roaming across different IP subnets. However, the mechanisms defined in MIP may cause undesired connection disruption or packet loss which will significantly degrade the quality of real-time applications. It is important yet challenging to support researches on seamless handoff management. To achieve seamless handoff, we propose a unified scheme to address application quality degradation. Our main contribution is the concept and implementation of utilizing buffering and resending method. It helps keep the end-to-end delay of real-time traffic flow in an acceptable value and eliminates the packet loss for accomplishing seamless handoff. The NS-2 simulation results also show that our proposed scheme can maintain application quality effectively for layer-2 and layer-3 handoff of a mobile node.
\end{abstract}

Keywords: IEEE 802.11, Mobile IP Protocol, Roaming, Seamless Handoff

\section{INTRODUCTION}

Following the IEEE 802.11 standard[1], the wireless devices can switch the connection from on to another between the linked base stations. Due to the switching, the wireless devices secure the best connection quality to the base stations. The procedure of layer- 2 handoff at present is hard handoff. It means the wireless devices have to disconnect with the original base station first, and then connect with the new one after authentication and association.

Mobile IP [2] protocol allows wireless equipment to move between different IP subnets and to maintain the connection with the Internet. When a wireless mobile node moves into another subnet, it may process layer-3 handoff following Mobile IP protocol (among different IP subnets) besides the abovementioned layer-2 handoff (among different base stations). Layer-3 handoff ensures that Internet application session such as World Wide Web, File Transfer or E-mail will not be disconnected.
Fig. 1 illustrates the general structure of wireless networks with layer-2 handoff and layer-3 handoff. The wireless environment is composed of wireless devices, base stations and a distribution network system (DS). Every base station forms a basic service set (BSS) with certain signal quality and range limited by its radio wave. Base stations provide a gate for wireless devices to access the Internet, and manage packets transmission or reception applying wireless devices. Different base stations are connected by a distribution wired network system (whether they are routers, switches or hubs), and they share the same basic service set identity. This extended service area is called an extended service set (ESS). It is analogous to the expanded service range of the base station.

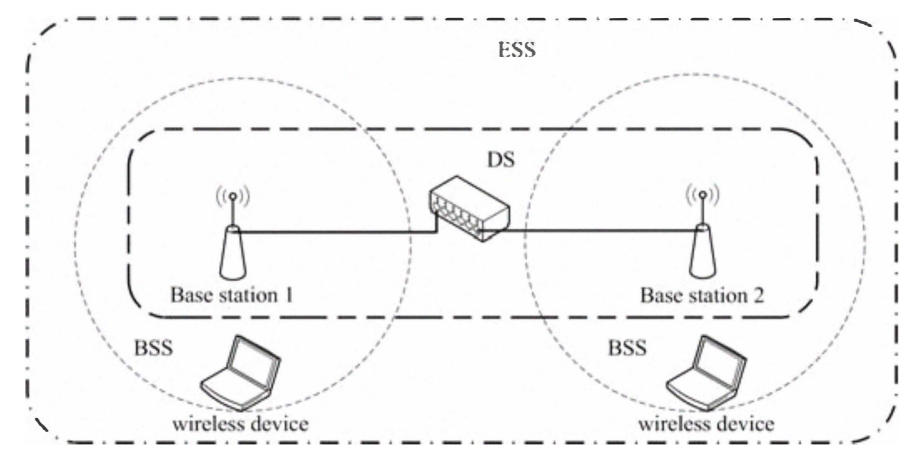

Figure 1. IEEE 802.11 BSS, ESS and DS

There are assumptions and theories proposed in the literature. Some of these introduce location information into mobile devices [3]. Some of these assumptions are realistic to existing IEEE 802.11 wireless environment [4]. For example, use layer-2 signal to trigger layer-3 activity [5] - [7], or add some layer-3 functionality into the base station (layer-2 device) For these cannot be performed with existing wireless devices, we can only hope for a more modern version of device drivers or firmware from the extensive wireless device manufacturers. Some of these use multiple wireless network interfaces or technologies [8] - [12] to reduce handoff latency. Furthermore, there are the compatibility problems with the existing devices. For example, using layer-2 signal to trigger layer-3 handoff or adding layer-3 functions into layer- 2 device will destroy the independence of network model and will be more complex for future applications. 
In this paper we propose a scheme to avoid the packet losses of handoff of wireless mobile devices using a single wireless network interface for deployed wireless environment (IEEE $802.11 \mathrm{~b} / \mathrm{g}$ ). It is not necessary to change software drivers (wireless mobile nodes) applying this method. Besides, it follows existing standards and protocols as much as possible.

\section{PROPOSED SEAMLESS HANDOFF SCHEME}

A wireless network interface following IEEE $802.11 \mathrm{~b} / \mathrm{g}$ standard applies hard handoff and forward mode at present. The interface uses only one radio channel to connect with one base station. So, when a handoff takes place, the interface has to disconnect with the current base station first; then establishes connection with a new base station.

There is a mechanism defined in the IEEE $802.11 \mathrm{~b} / \mathrm{g}$ standard for measuring the radio signal strength of the current associated base station received by a mobile node, which is called Receive Signal Strength Indicator (RSSI). Another mechanism is the signal to noise ratio (SNR) of the current associated base station received by a mobile node. Once the RSSI or SNR of the link between the current associated base station and a mobile node drops below a threshold, a handoff is about to take place.

Currently, layer-2 handoffs in most off-the-shelf IEEE 802.11 wireless interfaces use a firmware mode with a full scanning approach. An empirical measurement shows that the active scanning latency could be in the range of 350 to 500 milliseconds [13]. Longer latency results in more packet losses. So, if a wireless interface uses firmware mode layer- 2 handoff, it is hard to improve the handoff latency for time critical applications. For example, the disruptions in a voice over IP application would be noticed if the "jitter" is above 50 milliseconds. Therefore, we need a modified wireless interface driver which can reduce handoff latency by itself or allows other software to control handoff procedures [14]. Namely, in order to satisfy time critical applications, we need a new scheme to manage mobile node's handoff procedures. To meet the demand, we propose a seamless handoff scheme.

\section{A. Design of Proposed Handoff Scheme}

We present a seamless handoff algorithm to avoid the packet loss during a mobile node handoff that uses a single wireless network interface. In this algorithm:

- When a mobile node receives beacons from its current associated base station, it resets an association timer. When the association timer idles or the link quality (RSSI or SNR) drops below a threshold, the mobile node uses a smooth and selective layer-2 handoff

- We apply base station buffering and resending approaches to eliminate the frame loss when a smooth and selective probing is in processing.

- After the smooth and selective probing procedure of the mobile node is completed, we use home agent buffering and resending approaches to avoid the packet loss, while obtaining authentication and association with the new base station and layer-3 Mobile IP handoff (if needed).

\section{B. Layer-2 Probing Processes}

\section{a) Smooth and Selective Layer-2 Handoff}

In the existing active scan handoff schemes, the radio channels are scanned one after another without interruption during the probing period. A mobile node can not transmit or receive any data frame in this period. Instead of consecutively scanning all radio channels, there are other methods. A mobile node takes a break from the radio channel scanning section after one radio channel is scanned, and switches back to normal data transmission section. After working in the normal data transmission mode for some time, the mobile node switches to the scanning section again searching other radio channels. When scanning is completed, the mobile node has the knowledge of the neighboring base stations. Then it can choose one base station with the best signal quality (RSSI) as the new base station. Fig. 2 shows the process of smooth and selective layer-2 handoff scheme.

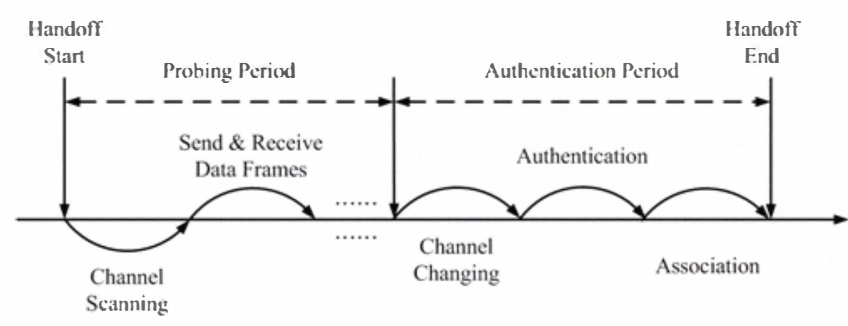

Figure 2. Smooth and Selective Layer-2 Handoff

The smooth and selective layer-2 handoff scheme has a longer handoff period than the traditional active scan scheme, but lower packet losses [15].

\section{b) Base Station Buffering and Resending}

The period of one radio channel scanning section in our smooth and selective layer-2 handoff scheme will be MinChannel-Time when no base station exists in this radio channel or Max-Channel-Time otherwise. The Min-Channel-Time is about $20 \mathrm{~ms}$ and the Max-Channel-Time is about $40 \mathrm{~ms}$ [13]. During this time, there may be frame losses. Therefore, we utilize base station buffering and resending to eliminate the frame losses.

For maintaining the frames, we block network interface queue, and delay the transmission, until the mobile node switches back to the normal data transmission section during the probing period. This method can eliminate all possible frame losses for upstream traffic.

- When a channel probing starts, the mobile node sends a BS_BUFF-Request message to the associated base station to ask for buffering frames destined to it during the channel probing section.

- The associated base station sends a BS_BUFF-Reply message back to the mobile node and starts buffering frames destined to the mobile node.

- After receiving BS_BUFF-Reply message sent by the associated base station, the mobile node starts channel probing. 
- When the timer of channel probing is expired, the mobile node sends a BS_BUFF_Replay-Request message to the associated $\bar{b}$ ase station asking for resending buffered frames in the base station while channel probing.

- The associated base station sends a BS_BUFF_Replay-Reply message back to the mobile node and starts resending buffered frames to the mobile node.

- When the mobile node receives the BS_BUFF_Replay-Reply message, it switches back to data transmission section.

\section{c) Mobility Agent Buffering and Resending}

After a mobile node finishes the radio channel probing, it makes a decision about choosing new associated base station. It then switches to the new radio channel and starts the authentication and association procedures. If the new base station belongs to a new subnet, it needs to process the MIP registration. Packets destined to the mobile node will be lost during these periods (layer- 2 authentication, association and layer-3 MIP handoff).

Whenever a wireless mobile node moves from home network to a foreign network or the reverse, the packets destined to this mobile node can be buffered by its home agent. Therefore, we can use home agent buffering and resending to avoid packet losses in those situations. If a mobile node uses route optimization [16] method when it moves into foreign networks, the packets will be routed direct through the foreign agent. We would like to extend this issue in our future works.

- After a wireless mobile node selects the new base station, it sends a layer-3 MIP HA BUFF-Request message to its home agent, and asks for buffering packets destined to it when it is in the process of layer- 2 authentication, association and layer-3 MIP handoff.

- The home agent sends a MIP_HA_BUFF-Reply message back to the mobile node and starts buffering packets destined to it.

- When receiving the MIP_HA_BUFF-Reply message sent by the home agent, the mobile node changes radio channel and starts authentication and association procedures with the new base station.

- After the mobile node has associated with a new base station, it sends a MIP_HA_BUFF_Replay-Request message (which is the same as MIPM̄H_Registration message but with different type) to the home agent asking for resending buffered packets.

- The home agent sends a MIPMH_Registration-Reply message back to the mobile node and starts resending buffered packets to the mobile node.

- When the mobile node receives the MIPMH_Registration-Reply message, it starts transmitting packets normally. The whole handoff process is completed at this moment.
For the packets transmitted by the mobile node, we block the queue of network interface, and hold those packets in this period till the mobile node finishes all handoff procedures. So, we can avoid all possible packet losses for upstream traffic. And the home agent buffers packets in individual queues differentiated by the home addresses of mobile nodes.

\section{PERFORMANCE EVAlUATION}

In this section, our proposed new handoff scheme is evaluated using "The Network Simulator ns-2.33" [17]. Channel probing latency, authentication and association latency and MIP handoff latency are compared with traditional and IPIAPP [18] - [20] handoff.

The simulation topology is shown in Fig. 3. Here, base stations (BS) 1 and 2 belong to the same IP subnet and are connected by a switch, while base station 3 resides in another IP subnet. These two different IP subnets and the subnet of correspondent node $(\mathrm{CN})$ are connected by three routers (R1, $\mathrm{R} 2$ and R3). Base stations 1 and 2 and router 1 create a local area network (LAN). We configure router 1 as the home agent (HA) for the mobile node (MN); and base station 3 is a foreign agent (FA). The correspondent node sends a CBR flow to the mobile node and vice versa.

The distance between two base stations is 460 meters. The transmitting distance between the wireless interface of the mobile node and the three base stations are 250 meters. So the coverage areas of these base stations overlap. The mobile node cannot receive packets from both base stations unless it is in the overlapping area. When the mobile node moves between the coverage areas of base stations 1 and 2 of the same IP subnet, this is a layer- 2 handoff. When the mobile node moves between the coverage areas of base stations 2 and 3 of different subnets, this is a layer- 3 handoff.

We set the data rate of wireless interface (IEEE 802.11) in our simulation to be $11 \mathrm{Mbits} / \mathrm{sec}$, the beacon interval of every base station is $100 \mathrm{~ms}$. We test each 3 scenarios for 10 times using different seeds, and calculate the average value of the simulation results.

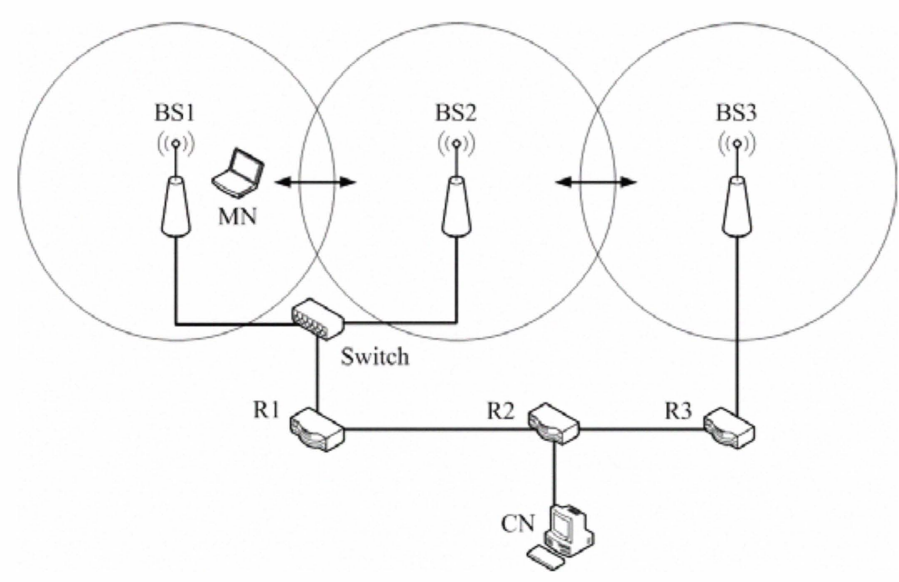

Figure 3. Simulation Topology

a) Average Number of Packet Loss during Handoff 
The average packet loss during handoff in the simulations of 3 scenarios using 10 different seeds in the traditional, IPIAPP and our proposed scheme are explained in TABLE 1.

TABLE I. AvERAgE NUMBER OF PACKET LOSS DURING HANDOFF

\begin{tabular}{|c|c|c|c|c|c|c|}
\hline \multirow{2}{*}{} & \multicolumn{3}{|c|}{$\begin{array}{c}\text { MN moves into } \\
\text { Foreign neIw ork }\end{array}$} & \multicolumn{3}{c|}{$\begin{array}{c}\text { MN moves back } \\
\text { 10 home network }\end{array}$} \\
\cline { 2 - 7 } & $\begin{array}{c}\text { Traditional } \\
\text { scheme }\end{array}$ & $\begin{array}{c}\text { IP-IAPP } \\
\text { scheme }\end{array}$ & $\begin{array}{c}\text { Proposed } \\
\text { scheme }\end{array}$ & $\begin{array}{c}\text { Traditional } \\
\text { scheme }\end{array}$ & $\begin{array}{c}\text { IP-IAPP } \\
\text { scheme }\end{array}$ & $\begin{array}{c}\text { Proposed } \\
\text { scheme }\end{array}$ \\
\hline Layer-2 handoff & 22.7 & 21.9 & 0.0 & 21.2 & 21.4 & 0.0 \\
\hline $\begin{array}{c}\text { Layer-3 handoff } \\
\text { LAN router as HA }\end{array}$ & 54.4 & 40.6 & 0.0 & 46.3 & 23.4 & 0.0 \\
\hline $\begin{array}{c}\text { Layer-3 handofi } \\
\text { BS as HA }\end{array}$ & 46.7 & 39.6 & 0.0 & 50.0 & 23.0 & 0.0 \\
\hline
\end{tabular}

Observations:

- In our proposed handoff scheme, we utilize base station buffering and resending to avoid frame loss in channel probing period. The mobile agent buffering and resending are used to avoid packet loss in authentication and mobile IP binding update periods. So, there is no packet loss in the simulations of the 3 scenarios using our proposed scheme.

- The layer-2 handoff latency is shorter than layer-3 handoff latency. For a layer-2 handoff does not need to process MIP binding update, the average packet loss number of a layer- 2 handoff is less than that in layer-3 handoffs.

- Layer-3 handoff latency is related to the time that a mobile node receives the Mobile IP advertisement message of the network mobility agent after it moves into this network. So the packet loss number of a layer-3 handoff in our simulation varies from 22 (IPIAPP) to 71 (traditional) packets.

- The IP-IAPP scheme includes the function of mobility agent to the base station. The base station can identify the movement of mobile nodes. If it is a layer-3 handoff, it establishes an IP tunnel from HA to FA for the mobile node actively. So, the packet loss number of IP-IAPP layer-3 handoff in our simulation is smaller than that in the traditional scheme.

\section{b) End-to-End Delay}

We examine the traditional and our proposed scheme for all 3 scenarios using the same parameters (Seed $=1$, MinChannel-Time $=20$, Max-Channel-Time $=40 \mathrm{~ms}$ and Normaltransmitting-section $=40 \mathrm{~ms}$ ). The simulation results of end-toend delay of CBR traffic flow are shown in Fig. 7, Fig. 8 and Fig. 9.

\section{Observations:}

- A mobile node starts handoff procedure after it disconnects with its associated base station in the traditional scheme. During the handoff, the mobile node cannot receive or transmit packets, thus suffers packet loss in the meantime. We learn that there are 2 broken regions in every scenario resulting from packet losses in Fig. 4, Fig. 5 and Fig. 6.

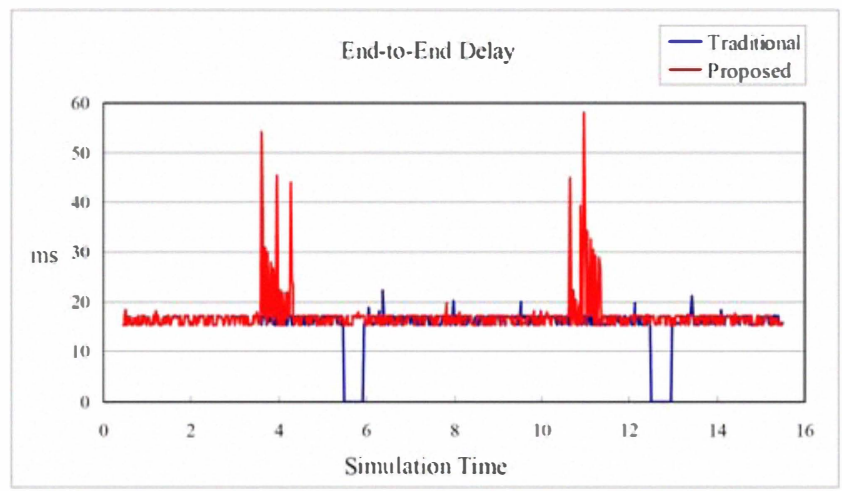

Figure 4. End-to-End Delay of Layer-2 handoff

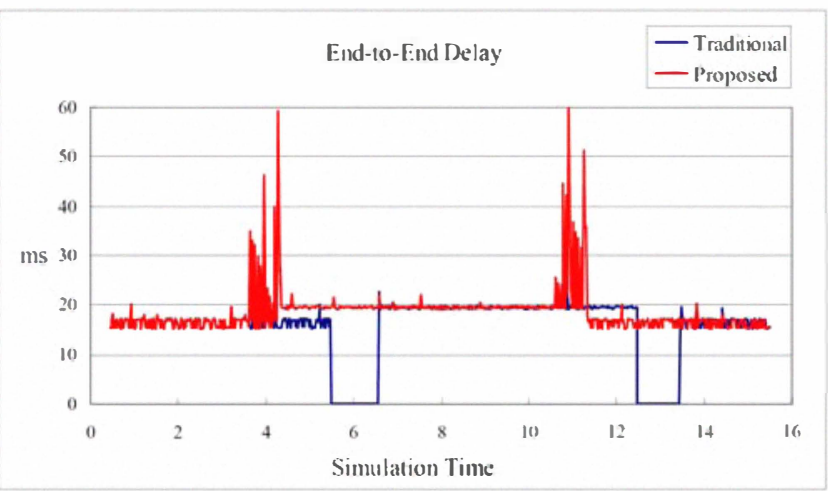

Figure 5. End-to-End Delay of Layer-3 handoff, LAN Router as $H A$

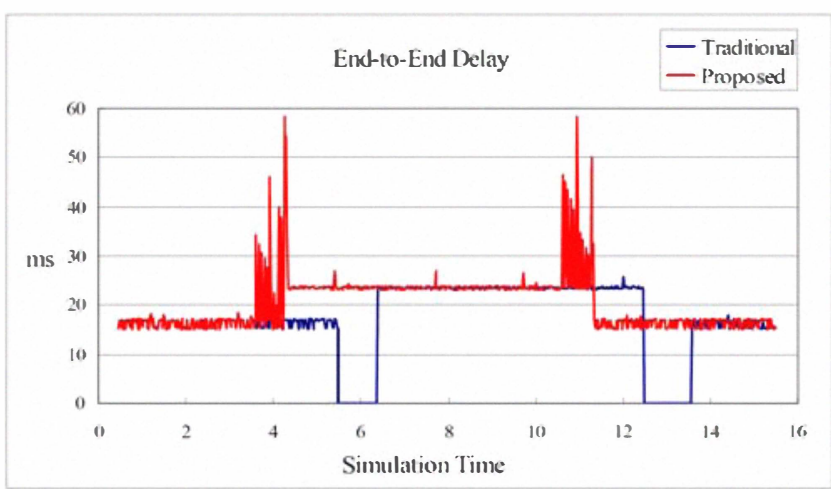

Figure 6. End-to-End Delay of Layer-3 handoff $B S$ as $H A$

- In our proposed handoff scheme, a mobile node starts the handoff procedure when the RSSI of received beacon from the associated base station drops below the threshold. It starts handoff earlier than loses connection with the associated base station (the jitter of proposed handoff scheme is earlier than the broken region in the traditional scheme). 
- In a layer-2 handoff, we realize there is an end-to-end delay after a layer- 2 handoff procedure. It is similar to that before the handoff in Fig. 4.

- In layer-3 handoffs, the end-to-end delay is longer when a mobile node moves into a visited network as shown in Fig. 5 and Fig. 6.

- The large part of end-to-end delay in the handoff periods is the Max-Channel-Time $(40 \mathrm{~ms})$ as shown in Fig. 4, Fig. 5 and Fig. 6.

\section{CONCLUSIONS AND FUTURE WORK}

In order to support seamless handoff for a mobile node, the end-to-end delay and the packet losses of a real-time traffic flow have to be reduced to an acceptable extent. In this paper, we proposed a new handoff scheme to achieve this requirement

The proposed handoff scheme employs a smooth channel probing procedure. A mobile node changes the radio channel back to the original associated one during the probing and starts a normal transmission section for a short period. It then approaches another channel to decrease the frame delay in the base station (which is waiting for all channels probing in the traditional handoff method) and to reduce the end-to-end delay of a real-time traffic flow. In the channel probing period, the proposed scheme utilizes base station buffering and resending to avoid frame losses. Also, in authentication, association and mobile IP binding update periods, the proposed scheme engages mobility agent buffering and resending to avoid packet losses.

We use CBR traffic to simulate a VoIP traffic flow in layer- 2 and layer- 3 handoff situations. The results show that the proposed scheme can eliminate the packet losses in a layer- 2 and a layer- 3 handoff. Besides, the maximum endto-end delay in a layer- 3 handoff period is controlled in an acceptable extent.

Future work on this subject may include:

- Use buffering and resending method on a foreign agent to optimize the routes when a mobile node moves into visited networks.

- Investigate on the speed of mobile nodes related to wireless deployed topology and link threshold to activate handoff procedure.

- Achieve seamless handoff for intensive network loading (more mobile nodes or combined with other traffic flows)

\section{REFERENCES}

[1] IEEE Std 802.11 (MAC) and Physical Layer (PHY) Specifications," June, 2007.

[2] C. Perkins, Ed., "IP Mobility Support for IPv4," Internet Engineering Task Force (IETF), RFC 3344, August, 2002.

[3] CheolHee Lee, SungHoon Seo, and JooSeok Song, "An Indoor Tracking-based Handoff Mechanism for VoIP Applications in IEEE 802.11 WLANs," 4th International Conference on Information and Automation for Sustainability, Pages: 324-329, December, 2008.
[4] Emil Ivov, Julien Montavont and Thomas Noel, "Thorough Empirical Analysis of the IETF FMIPv6 Protocol over IEEE 802.11 Networks," IEEE Wireless Communications, Volume 15, Issue 2, Pages: 65-72, April, 2008.

[5] Albert Hasson, Neco Ventura and Sven Shepstone, "Mobile IP Movement Detection Optimizations in 802.11 Wireless LANs," 1st International Symposium on Wireless Communication Systems, Pages: 393-397, September, 2004.

[6] K. El Malki, Ed., "Low Latency Handoffs in Mobile IPv4," Internet draft, draft-ietf-mobileip-lowlatency-handoffs-v4-11.txt, work in progress. June, 2007.

[7] Srikant Sharma, Ningning Zhu and Tzi-cker Chiueh, "Low-Latency Mobile IP Handoff for Infrastructure-Mode Wireless LANs," IEEE Journal on Selected Areas in Communications, Volume 22, Issue 4, Pages: 643-652, May, 2004.

[8] Ashutosh Dutta, David Famolari, Subir Das, Yoshihiro Ohba, Victor Fajardo, Kenichi Taniuchi, Rafael Lopez and Henning Schulzrinne, "Media-Independent Pre-Authentication Supporting Secure Interdomain Handover Optimization," IEEE Wireless Communications, Volume 15, Issue 2, Pages: 55-64, April, 2008

[9] George Lampropoulos, Apostolis K. Salkintzis and Nikos Passas, "Media-Independent Handover for Seamless Service Provision in Heterogeneous Networks," IEEE Communications Magazine, Volume 46, Issue 1, Pages: 64-71, January, 2008.

[10] Ok Sik Yang, Seong Gon Choi, Jun Kyun Choi, Jung Soo Park and Hyoung Jun Kim, "A Handover Framework for Seamless Service Support between Wired and Wireless Networks," The 8th International Conference on Advanced Communication Technology, Volume 3, Pages: 1791-1796, February, 2006.

[11] Philippe Chiron, Eric Njedjou, Pierrick Seite, Karine Gosse, Eric Melin and Pierre Roux, "Architectures for IP-Based Network-Assisted Mobility Management Across Heterogeneous Networks," IEEE Wireless Communications, Volume 15, Issue 2, Pages: 18-25, April, 2008.

[12] Quoc-Thinh Nguyen-Vuong, Nazim Agoulmine and Yacine GhamriDoudane, "Terminal-Controlled Mobility Management in Heterogeneous Wireless Networks," IEEE Communications Magazine, Volume 45, Issue 4, Pages: 122-129, April, 2007.

[13] Arunesh Mishra, Minho Shin and William Arbaugh, "An Empirical Analysis of the IEEE 802.11 MAC Layer Handoff Process," $A C M$ SIGCOMM Computer Communication Review, Volume 33, Issue 2, Pages: 93-102, April, 2003.

[14] Hanane Fathi, Ramjee Prasad and Shyam Chakraborty, "Mobility Management for VoIP in 3G Systems: Evaluation of Low-Latency Handoff Schemes," IEEE Wireless Communications, Volume 12, Issue 2 , Pages: 96-104, April, 2005.

[15] Yong Liao and Lixin Cao, "Practical Schemes for Smooth MAC Layer Handoff in 802.11 Wireless Networks," International Symposium on a World of Wireless, Mobile and Multimedia Networks, Pages: 181-190, June, 2006.

[16] Charles E. Perkins and David B. Johnson, "Route Optimization in Mobile IP," Internet Draft, draft-ietf-mobileip-optim-11.txt, September, 2001

[17] The Network Simulator - ns-2, http://www.isi.edu/nsnam/ns/.

[18] Chun-Ting Chou and Kang G. Shin, "An Enhanced Inter-Access Point Protocol for Uniform Intra- and Inter-subnet Handoffs," IEEE Transactions on Mobile Computing, Volume 4, Issue 4, Pages: 321-334, July-August, 2005.

[19] IEEE Std 802.11FTM-2003, "IEEE Trial-Use Recommended Practice for Multi-Vendor Access Point Interoperability via an Inter-Access Point Protocol Across Distribution Systems Supporting IEEE 802.11 Operation," July, 2003.

[20] Ioanna Samprakou, Christos Bouras and Theodore Karoubalis, "Fast IP Handoff Support for VoIP and Multimedia Applications in 802.11 WLANs," Sixth IEEE International Symposium on a World of Wireless Mobile and Multimedia Networks, Pages: 332-337, June, 2005. 